\title{
Gravity mode offset and properties of the evanescent zone in red-giant stars
}

\author{
S. Hekker ${ }^{1,2}$, Y. Elsworth ${ }^{2,3}$, and G. C. Angelou ${ }^{1,2}$ \\ ${ }^{1}$ Max-Planck-Institut for Solar System Research, Justus-von-Liebig-Weg 3, 37077 Göttingen, Germany \\ e-mail: Hekker@mps.mpg.de \\ 2 Stellar Astrophysics Centre, Department of Physics and Astronomy, Aarhus University, Ny Munkegade 120, 8000 Aarhus C, \\ Denmark \\ ${ }^{3}$ School of Physics and Astronomy, University of Birmingham, Birmingham B15 2TT, UK
}

Received 29 May 2017 / Accepted 13 December 2017

\begin{abstract}
Context. The wealth of asteroseismic data for red-giant stars and the precision with which these data have been observed over the last decade calls for investigations to further understand the internal structures of these stars.

Aims. The aim of this work is to validate a method to measure the underlying period spacing, coupling term, and mode offset of pure gravity modes that are present in the deep interiors of red-giant stars. We subsequently investigate the physical conditions of the evanescent zone between the gravity mode cavity and the pressure mode cavity.

Methods. We implement an alternative mathematical description compared to what is used in the literature to analyse observational data and to extract the underlying physical parameters that determine the frequencies of mixed modes. This description takes the radial order of the modes explicitly into account, which reduces its sensitivity to aliases. Additionally, and for the first time, this method allows us to constrain the gravity mode offset $\epsilon_{g}$ for red-giant stars.

Results. We find that this alternative mathematical description allows us to determine the period spacing $\Delta \Pi$ and the coupling term $q$ for the dipole modes within a few percent of values found in the literature. Additionally, we find that $\epsilon_{g}$ varies on a star-by-star basis and should not be kept fixed in the analysis. Furthermore, we find that the coupling factor is logarithmically related to the physical width of the evanescent region normalised by the radius at which the evanescent zone is located. Finally, the local density contrast at the edge of the core of red-giant branch models shows a tentative correlation with the offset $\epsilon_{g}$.

Conclusions. We are continuing to exploit the full potential of the mixed modes to investigate the internal structures of red-giant stars; in this case we focus on the evanescent zone. It remains, however, important to perform comparisons between observations and models with great care as the methods employed are sensitive to the range of input frequencies.
\end{abstract}

Key words. asteroseismology - methods: data analysis - stars: interiors

\section{Introduction}

The long, near-uninterrupted, high-precision photometric timeseries data from the CoRoT and Kepler space missions now allow for the investigation of the internal structures of stars. One of these structure features in red-giant stars is the evanescent zone between the cavity in which oscillations are present with buoyancy as restoring force ( $g$-mode cavity) and the cavity in which pressure is the restoring force ( $p$-mode cavity). The location, shape, and width of this evanescent zone may all play a role in the coupling between these cavities and the characteristics of the observed dipole modes (modes with degree $l=1$ ), which have a mixed pressure-gravity nature (Takata 2016a,b; Mosser et al. 2017). We note here that generally the dipole modes, rather than quadrupole modes (modes with degree $l=2$ ) are used to investigate the interior conditions in stars. This is due to two phenomena: the coupling is weaker at higher degrees, leading to very small amplitudes of the modes with a significant $\mathrm{g}$ component, and the spacing between the mixed components reduces as a function of degree, making quadrupole mixed modes and their period spacings harder to resolve. In the remainder of the paper we discus dipole modes, in all cases where no degree is indicated.

The underlying characteristics of the gravity part of the mixed modes are the asymptotic period spacing $(\Delta \Pi)$, the coupling factor $(q)$, and an offset $\left(\epsilon_{g}\right)$. The asymptotic period spacing is the period spacing between pure gravity modes ( $g$ modes) in the limit where the degree of the mode is much lower than the radial order $n$ (i.e. $l \ll n$ ). The spacing in period between individual mixed modes is in theory always smaller than the asymptotic value due to the coupling with a pressure mode. The coupling factor provides insight into the strength of the coupling between the $g$-mode cavity and the $p$-mode cavity, with $q=0$ for no coupling and $q=1$ indicating maximum coupling. The parameter $\epsilon_{g}$ is a phase term accounting for the behaviour near the turning points of modes (e.g. Hekker \& Christensen-Dalsgaard 2017).

Various approaches have been employed to determine the parameters of the mixed modes. For a subset of known red giants, Bedding et al. (2011) derived the most prominent period spacing by taking the power spectrum of the power spectrum for dipole modes, where the mode frequencies were expressed in period and the amplitude of the power spectrum was set to zero 
in regions not containing $l=1$ modes. Using this period spacing they presented period-échelle diagrams. In such diagrams frequency $(v)$ is shown as a function of period $(\Pi)$ modulo period spacing $(\Delta \Pi)$; see, for example, panel $E$ of Fig. 1 . The frequencies of the mixed modes in consecutive acoustic radial orders are stacked on top of each other and show a typical "S-shape". The value of $\epsilon_{g}$ determines the absolute position of the "S-shape" pattern in the period-échelle diagram, while $q$ determines the steepness of the central segments; a shallow transition in the case of strong coupling and a steep transition in the case of weak coupling.

Mosser et al. (2012b) presented the asymptotic expansion for the frequencies $(v)$ of mixed modes based on Unno et al. (1989):

$v=v_{n_{p}, l}+\frac{\Delta v}{\pi} \arctan \left[q \tan \pi\left(\frac{1}{\Delta \Pi_{l} v}-\epsilon_{g}\right)\right]$,

where $v_{n_{p}, l}$ is the frequency of the pressure mode (with radial order $n_{p}$ and degree $l$ ) with which the gravity modes are coupled, $\Delta \Pi_{l}$ is the asymptotic period spacing of modes with degree $l$, and $\Delta v$ is the large frequency separation between modes of the same degree and consecutive radial orders of acoustic modes. One commonly adopted assumption is to take $\epsilon_{g}$ as a fixed value: either zero or one half depending on the definitions used. By fixing $\epsilon_{g}$ it is possible to determine the period spacing and coupling strength in an iterative manner. This formulation has successfully been applied in many cases (e.g. Mosser et al. 2014; Buysschaert et al. 2016). In fact, Buysschaert et al. (2016) were the first to leave $\epsilon_{g}$ as a free parameter in Eq. (1) and concluded that this enables a more robust analysis of both the asymptotic period spacing and the coupling factor. However, their method left $\epsilon_{g}$ illdefined with a large confidence interval (e.g. Buysschaert et al. 2016).

Datta et al. (2015) developed an automated way to find the optimal value of $\Delta \Pi_{l}$ by measuring the alignment of the "Sshape" and the symmetry in the period-échelle diagram, that is, implicitly assuming a fixed value for $\epsilon_{g}$. Furthermore, to extract reliable period spacings they used a Monte Carlo approach whereby period spacings were computed for 10000 realisations of the data, for which the frequencies were randomly perturbed within their uncertainties. The distribution of the $\Delta \Pi_{l}$ results of each perturbation could then be used to compute a value with uncertainties for each discrete solution of $\Delta \Pi_{l}$ as well as the probability of the solution. Hence, in their analysis, multiple results of the period spacing for a particular star with their probabilities were presented.

Following this, Mosser et al. (2015) realised that the observed and asymptotic period spacings can be related through the ratio $(\zeta)$ between the kinetic energy in the radiative cavity and the total kinetic energy (e.g. Goupil et al. 2013; Deheuvels et al. 2015). This relation allowed Mosser et al. (2015) to use $\zeta$ to compute stretched periods of mixed modes, where the modes in the stretched period-échelle diagram line up along vertical ridges. Vrard et al. (2016) used this concept to develop an automated tool to compute gravity period spacings for over 6100 red giants observed with the Kepler telescope. This method, using $\zeta$, is particularly powerful as it requires only an approximate determination of $\Delta v, \Delta \Pi_{1}$, and the frequency position of the dipole pressure modes. Moreover, this method is applied to the full power spectrum and does not require knowledge of the frequencies of individual modes.

Mosser et al. (2017) subsequently investigated the coupling factors, $q$, of thousands of red giants, with the intention of providing physical constraints on the regions surrounding the radiative core and the hydrogen-burning shell. They found that weak coupling is present in only the most evolved stars on the red-giant branch. Larger coupling factors are measured at the transition between subgiants and giants as well as in core helium burning $(\mathrm{CHeB})$ stars.

An alternative mathematical description that is consistent with Eq. (1) has been proposed by Christensen-Dalsgaard (2012), and developed further by Jiang \& Christensen-Dalsgaard (2014), Cunha et al. (2015), and Hekker \& Christensen-Dalsgaard (2017) to compute the theoretical frequencies of mixed modes. This formalism has so far been used to compute frequencies of mixed dipole modes for models. Here, we investigate the performance of this formalism in determining $\Delta \Pi, q$, and $\epsilon_{g}$ when applied to observed data. This method explicitly uses frequencies as well as the value of the radial order of mixed modes and has the advantage that it can be applied to models (with frequencies computed in an independent way) as well as to observational data. This allows us to investigate both the proximity of the period spacings computed from individual mixed-mode frequencies and from the integral of the Brunt-Väisälä frequency, and the physical conditions of the evanescent zone connected with the coupling term. Additionally, we comment on the physical meaning of $\epsilon_{g}$ and what the impact is of choosing a different set of frequencies to derive the period spacing.

\section{Method}

The formalism proposed by Christensen-Dalsgaard (2012) is as follows:

$\Pi_{n l}=\frac{1}{v_{n l}}=\Delta \Pi_{l}\left[|n|+\epsilon_{g}+\frac{1}{2}-\frac{\Phi\left(v_{n l}\right)}{\pi}\right]$,

where $|n|$ is the absolute value of the numerical radial mode order (see Sect. 2.2). Additionally, $\Phi\left(v_{n l}\right)$ satisfies

$\tan \Phi\left(v_{n l}\right)=q \cot \left(\pi\left(\frac{v_{n l}}{\Delta v}-\epsilon_{p l}\right)\right)$,

where we assume that $\Delta v$ has the same value as obtained from a linear fit through the radial modes, that is, a typical way to extract it from the frequency spectra, and $\epsilon_{p l}$ is an offset for the acoustic modes of degree $l$ (see Sect. 5.4 for more details).

\subsection{Implementation}

To apply the formalism outlined here to observed dipole frequencies we start by supplying the algorithm with an initial estimate of $\Delta \Pi_{l}$. We then compute the radial orders of the oscillation modes as per Eq. (7) and apply a $\chi^{2}$ fit procedure to Eq. (2). Here, we allow $|n|$ to vary by an integer and keep $\Delta v$ fixed to find the values of $\Delta \Pi, q, \epsilon_{g}$, and $\epsilon_{\mathrm{p} 1}$ that give a best fit to the observed frequencies of modes with a particular degree $(l)$ and azimuthal order $(m)$. This is based on the fact that for slowly rotating red giants we have computed the frequency of the $m=0$ component of modes with $m \neq 0$ using the description by Mosser et al. (2012a). To obtain the best fit with the lowest $\chi^{2}$ we use a grid of initial estimates of $\Delta \Pi$ ranging for red-giant branch stars from 50 to $100 \mathrm{~s}$ in steps of $0.01 \mathrm{~s}$ and for low-mass core-helium-burning stars from 170 to $360 \mathrm{~s}$ in steps of $0.1 \mathrm{~s}$. In this way we obtain for each initial $\Delta \Pi$ the radial order of the modes and a computed value for $\Delta \Pi, q, \epsilon_{g}$, and $\epsilon_{p 1}$ as well as a measure of the goodness of fit through the $\chi^{2}$ value. We note here that we have defined $\epsilon_{p 1}$ to have a value between 0.5 and 1.5 similar to $\epsilon_{p 0}$. 
In this goodness of fit, we have to account for the fact that we expect a larger number of gravity modes $\left(N_{v_{g}}\right)$ in a $\Delta v$ interval for lower values of $\Delta \Pi$ (Mosser et al. 2012b):

$N_{v_{g}} \cong \frac{\Delta v}{\Delta \Pi v_{\max }^{2}}$,

where $v_{\max }$ is the frequency of maximum oscillation power.

To incorporate uncertainties in the observed frequencies, as well as correlations between different parameters, we use a Monte Carlo approach with 100 iterations to perturb the observed frequencies randomly within their uncertainties. We apply the described method for each set of perturbed frequencies.

We note here that this formalism does not take into account effects of glitches, that is, sudden internal structure changes visible as variations in the oscillation frequencies and thus also period spacings (described in detail by Cunha et al. 2015, in the case of buoyancy glitches). As stated by Cunha et al. (2015), buoyancy-glitch-induced variations occur on the redgiant branch only at the luminosity bump, and after the red-giant branch only in the early phases of helium core burning and at the beginning of helium-shell burning. Hence, we expect that for many stars it is not necessary to perform a glitch analysis in order to extract period spacings.

\subsection{Radial order}

Fundamental to the formalism discussed here is our knowledge of the radial order of every dipole feature that is present. Following the asymptotic analysis, the period of a pure gravity mode can be expressed as

$\Pi_{n 1}=\Delta \Pi\left(\left|n_{g}\right|+\epsilon_{g}+1 / 2\right)$.

From this we can estimate the absolute value of the gravity mode order $n_{g}$ as

$\left|n_{g}\right| \cong \frac{1}{\Delta \Pi v_{n 1}}$,

where we neglect the $\epsilon_{g}+1 / 2$ as $\left|n_{g}\right| \gg \epsilon_{g}+1 / 2$. The final estimate of the radial order $n$ of a specific frequency of a mixed mode is a combination of the pure gravity radial order and the pure pressure radial order $\left(n_{p}\right)$ and can be computed as:

$n=n_{g}+n_{p} \cong \frac{-1}{\Delta \Pi v_{n 1}}+\left(\frac{v_{n 1}}{\Delta v}-\epsilon_{p 1}\right)$,

where we used the general convention that gravity mode orders are indicated with negative values. We note that $\Delta v$ and $\epsilon_{p 0}$ are computed from a linear fit through the radial frequencies and that a first estimate of $\epsilon_{p 1}$ is obtained using the correction for the degree according to $\epsilon_{p 1} \approx \epsilon_{p 0}+1 / 2$ (see also Sect. 5.4). Combined with the requirement that the radial order of each mode should be an integer and that $n$ should increase with frequency, we can compute $n$ provided that all other parameters are known. To obtain the radial mode orders of modes with $m \neq 0$ we estimate the rotational splitting using a Lorentzian profile as proposed by Mosser et al. (2012a) to identify the frequency of the underlying unsplit mode and use that frequency to compute the radial order in the same way as outlined above.

\section{Data}

In this section, we indicate the data to which we apply the method outlined above. These data comprise theoretical models and observational data of both red-giant branch (RGB) stars and core helium burning $(\mathrm{CHeB})$ stars.

\subsection{Red-giant branch stars}

We used the three RGB models described by Datta et al. (2015) to test the application of the formalism discussed in this manuscript. The models by Datta et al. (2015) are $1 M_{\odot}$ models at different stages of hydrogen shell burning computed using the MESA stellar evolution code (Paxton et al. 2011). These models were chosen because they are computed in a manner that is independent of the development of the formalism discussed here and $\Delta \Pi$ has been provided. From the models, we prepared sets of frequencies that mimic "observational" data by selecting modes in three frequency ranges with 5, 7, and 9 radial orders centred around $v_{\max }$. In this frequency range we kept either all frequencies, or we selected modes with normalised inertias (with respect to radial-mode inertias) such that on average we have 5 modes per (acoustic) radial order. In the analysis we neglected the fact that we know the radial orders of these oscillation modes. We assumed an uncertainty of $0.008 \mu \mathrm{Hz}$ on all dipole frequencies. This value is approximately the frequency resolution of the approximately 4-yr long timeseries of Kepler data. Throughout the paper we show the results obtained for the mode sets with all dipole modes in a $5 \Delta v$ wide frequency range.

Additionally, we applied our method to frequencies from stars observed by Kepler. We used frequencies for the sample of stars presented by Datta et al. (2015) and Corsaro et al. $(2015)^{1}$, as well as KIC 4447888 (Di Mauro et al. 2016). Two of the three stars analysed by Datta et al. (2015) are part of the sample analysed by Corsaro et al. (2015). We used both sets of data as the frequency values have been determined independently. In total, 21 stars were treated, with $\Delta \Pi$ values ranging from 68.5 to $90 \mathrm{~s}$ (see also Fig. A.1 for a visual representation of the distribution of dipole frequencies).

\subsection{Core helium burning stars}

In the last few years several studies (e.g. Bossini et al. 2015, 2017; Constantino et al. 2015; Lagarde et al. 2016) have investigated the physics that needs to be included to remedy the discrepancy between $\Delta \Pi$ values of $\mathrm{CHeB}$ stars derived from observations (e.g. Mosser et al. 2014) and from models with standard physics included. In this work we use the models described by Constantino et al. (2015) who computed $1 M_{\odot}$ solar metallicity $\mathrm{CHeB}$ models using the MONSTAR stellar evolution code (Lattanzio 1986; Campbell \& Lattanzio 2008; Constantino et al. 2014). These authors apply different core-mixing schemes at different phases of core helium burning, that is, just after the helium flash (or non-degenerate onset of He-core burning) all the way to exhaustion of helium in the core. We applied our method to the models in Figs. 9, 10, 12-14, 16, and 17 of Constantino et al. (2015), which were provided by the authors. Table 1 provides some basic information about the models. We use "regular", "irregular", "regular/spiky" and "semi-regular" to classify the observed period spacing $(\Delta P)$ of the models as a function of frequency. This classification is determined from a visual inspection of the $\Delta P$ versus frequency figures presented by Constantino et al. (2015). We classify a star as regular when $\Delta P$ (relatively) smoothly approaches minima at the pressure-dominated dipole modes and maxima at the radial modes (see e.g. the orange curve in the top panel of Fig. 12 of Constantino et al. 2015). When such

\footnotetext{
1 We increased the uncertainties on the frequencies as provided by Corsaro et al. (2015) by a factor of three, as it was shown that these were underestimated by roughly that factor (Corsaro et al., erratum in prep.).
} 
Table 1. Core helium burning models from Constantino et al. (2015) used in the current work.

\begin{tabular}{rllllc}
\hline \hline Fig. \# & Colour & $\Delta \Pi$ & Our work & Regularity $\Delta P$ & $\Delta \Pi_{\text {this work }}$ \\
\hline 9 & Black & $240 \mathrm{~s}$ & CHeBmodel 0 & Irregular* & $\sim 241 \mathrm{~s}$ \\
9 & Blue & $238 \mathrm{~s}$ & CHeBmodel 1 & Irregular* & $\sim 243 \mathrm{~s}$ \\
10 & Black & $247 \mathrm{~s}$ & CHeBmodel 2 & Irregular & - \\
10 & Blue & $247 \mathrm{~s}$ & CHeBmodel 3 & Regular* & $\sim 249 \mathrm{~s}$ \\
12 & Black & $252 \mathrm{~s}$ & CHeBmodel 4 & Irregular* & $\sim 253 \mathrm{~s}$ \\
12 & Orange & $253 \mathrm{~s}$ & CHeBmodel 5 & Regular* & $\sim 253 \mathrm{~s}$ \\
12 & Blue & $253 \mathrm{~s}$ & CHeBmodel 6 & Regular* & $\sim 253 \mathrm{~s}$ \\
13 & Blue & $253 \mathrm{~s}$ & CHeBmodel 7 & Regular* & $\sim 253 \mathrm{~s}$ \\
13 & Magenta & $314 \mathrm{~s}$ & CHeBmodel 8 & Regular* & $\sim 316 \mathrm{~s}$ \\
14 & Black & $278 \mathrm{~s}$ & CHeBmodel 9 & Irregular & - \\
14 & Cyan & $281 \mathrm{~s}{ }^{a}$ & CHeBmodel 10 & Regular/spiky & - \\
16 & Black & $273 \mathrm{~s}$ & CHeBmodel 11 & Semi-regular* & $\sim 274 \mathrm{~s}$ \\
16 & Orange & $271 \mathrm{~s}$ & CHeBmodel 12 & Regular/spiky & - \\
16 & Cyan & $264 \mathrm{~s}$ & CHeBmodel 13 & Regular/spiky & - \\
17 & Orange & $268 \mathrm{~s}$ & CHeBmodel 14 & Semi-regular* & $\sim 277 \mathrm{~s}$ \\
\hline
\end{tabular}

Notes. The first three columns give the figure number, the colour-code used in the figure, and the period spacing computed by Constantino et al. (2015). The last columns provide the identification that we use in this work, a comment concerning the regularity of the behaviour of the observed period spacing $(\Delta P)$ with frequency, and a rough value of our determined $\Delta \Pi$ for comparison purposes. The ${ }^{(*)}$ indicates models for which we reliably recover $\Delta \Pi$ and that are used in the further analysis in this paper. ${ }^{(a)}$ If the calculation includes only the region exterior to the chemical discontinuity then $\Delta \Pi=315 \mathrm{~s}$ (Constantino et al. 2015).

dips are not present, as is the case in the top panel of Fig. 9 of Constantino et al. (2015), we assign the classification "irregular". With "semi-regular" we indicate models for which $\Delta P$ shows dips as a function of frequency, but with a significant amount of irregular structure on top of that. With "regular/spiky", we refer to dips that are very narrow, such as the cyan curves in Figs. 14 and 16. We applied our method to all models that we have at our disposal.

The "observational" data were obtained from the models in the same way as per the RGB models described in the previous subsection. Additionally, we applied the procedure to a Li-rich star (KIC 5000307) in the red clump (Silva Aguirre et al. 2014).

\section{Results}

In this section, we present the results that we obtained with our method using the data described in the previous section. The results are presented in Tables 2 and 3.

We show an illustration of the results for a RGB star in Fig. 1. In this figure, histograms of the results with lowest $\chi^{2}$ per Monte Carlo iteration as a function of $\Delta \Pi, \epsilon_{g}, q$, and $\epsilon_{p 1}$ are shown in panels $\mathrm{A}, \mathrm{B}, \mathrm{C}$ and $\mathrm{D}$, respectively. Panel $\mathrm{E}$ shows a periodéchelle diagram based on the derived $\Delta \Pi$ value (value quoted in the $x$-axis label). Panel $\mathrm{E}$, in fact, consists of a repeated échelle diagram to enable better visualisation of the "S-shape". This is, in principle, possible for our solutions as we have $\epsilon_{g}$ as a free parameter. We show the results for a $\mathrm{CHeB}$ star in a similar way in Fig. 2.

We checked that the ratio of the uncertainty in $\epsilon_{g}\left(\sigma_{\epsilon_{g}}\right)$ to the relative uncertainty in $\Delta \Pi\left(\sigma_{\Delta \Pi} / \Delta \Pi\right)$ is roughly equal to $|n|$. This is generally satisfied for our results.

Below we discuss our results for both the RGB and $\mathrm{CHeB}$ stars and compare them with literature values and/or values obtained from models. These comparisons focus on period spacings and coupling factors as these parameters are available in the literature or can be computed in an independent way from the models.

\subsection{Red-giant branch stars}

The $\Delta \Pi$ results obtained in this work for all three models described by Datta et al. (2015) are in agreement with their values obtained from individual frequencies. The results are, however, most stable for Model 0 and less so for the other, more evolved models. This is due to the distribution of the dipole modes (see below) as well as the large absolute value of the radial order.

For 20 out of 21 observed stars (two stars, KIC009145955 and KIC010200377, are analysed twice with slightly different datasets) we find good agreement (better than 3\%) between the values of the period spacings obtained in this work and the results presented in the literature ${ }^{2}$. The relative differences are shown in Fig. 3. For one RGB star we find a somewhat larger discrepancy between the $\Delta \Pi$ value obtained in our work compared to the values obtained in the literature ( $\sim 4 \%$ difference): KIC 5866737. We discuss this star below in more detail. Additionally, we also compared our values with period spacings obtained by Vrard et al. (2016) for the 16 stars that we have in common. The differences in the period spacings in this comparison are in all cases well within $1 \%$.

For KIC 5866737 we find that the ratio of the uncertainty in $\epsilon_{g}\left(\sigma_{\epsilon_{g}}\right)$ to the relative uncertainty in $\Delta \Pi\left(\sigma_{\Delta \Pi} / \Delta \Pi\right)$ is not roughly equal to $|n|$. At the same time, we find a $\Delta \Pi$ value that is approximately $4 \%$ lower than obtained in the literature (see Fig. 3). KIC 5866737 is the most evolved star in our sample of observed stars, with dipole modes that are confined in a narrow range around the pressure-dominated mode. The coupling is expected to decrease for more evolved stars along the RGB and we conclude that KIC 5866737 roughly indicates the limit along the RGB at which the method discussed here can produce reliable results in terms of gravity mode parameters. We

\footnotetext{
2 We note that the results presented by Corsaro et al. (2015) are those obtained by Mosser et al. (2012b), and that for KIC 6144777 and KIC 7060732, we have used updated values of $\Delta \Pi=79.23 \mathrm{~s}$ and $\Delta \Pi=77.10 \mathrm{~s}$, respectively (Corsaro, priv. comm.).
} 

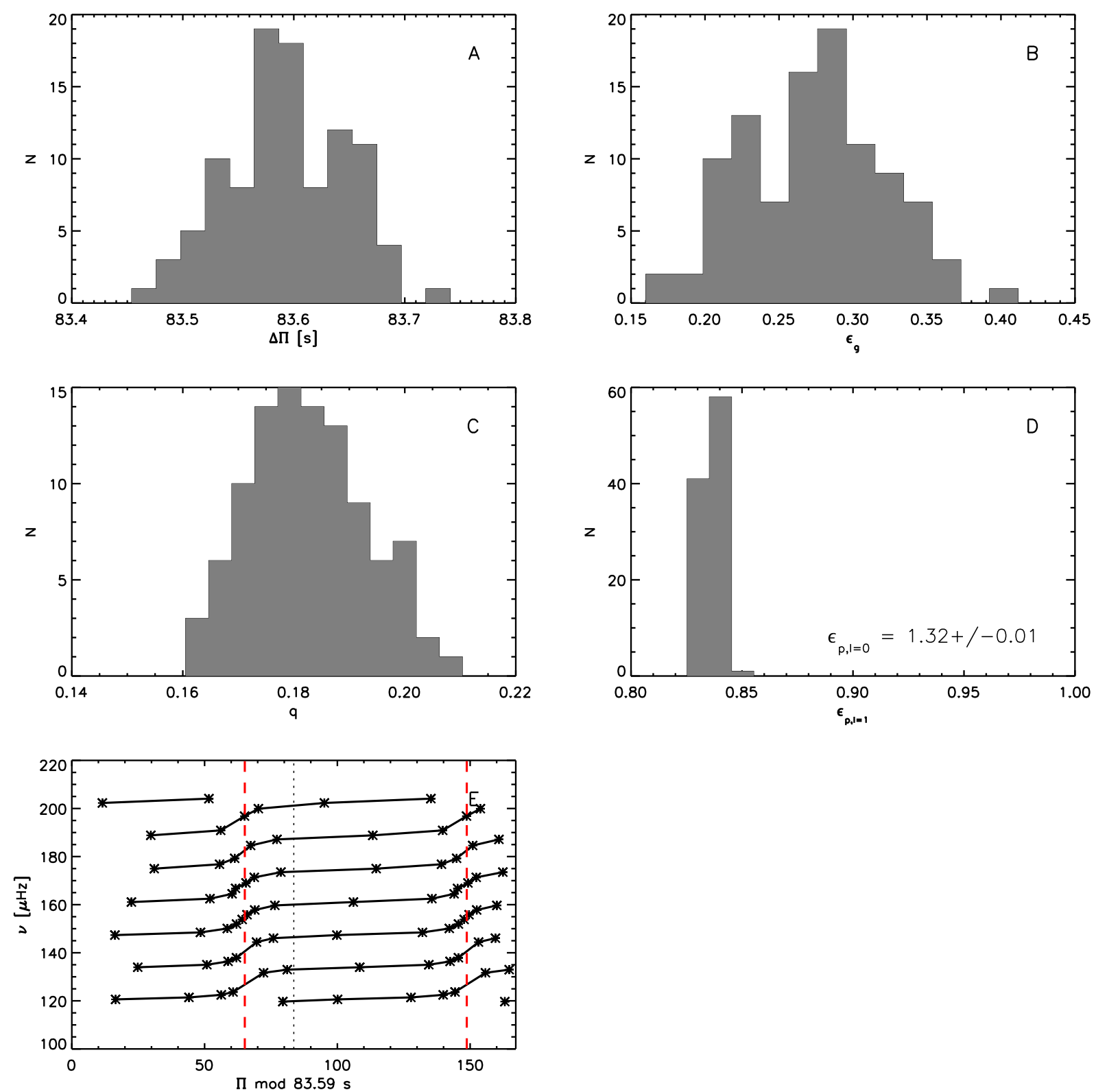

Fig. 1. Results for KIC 10123207 using the frequencies from Corsaro et al. (2015). Panels $A-D$ : histograms of $\Delta \Pi, \epsilon_{q}, q$, and $\epsilon_{\mathrm{p} 1}$ (see Introduction for the meaning of these parameters). The $\epsilon_{\mathrm{p} 0}$ value determined from radial modes is indicated in the legend of panel $D$. A period-échelle diagram using the $\Delta \Pi$ obtained in this work is shown in panel $E$. The vertical red dashed lines indicate $\left(\left(\epsilon_{g}+0.5\right) \bmod 1\right) * \Delta \Pi$, that is, the position of the most $g$-dominated modes according to the fitted values. We note that the period-échelle diagram is shown twice separated by the dotted vertical line.

note that for RGB Model $1(\Delta \Pi \approx 72$ s) and RGB Model 2 $(\Delta \Pi \approx 62 \mathrm{~s})$ the datasets that mimic observations also cause the method difficulties and fail in a number of cases.

In Fig. 4 the coupling factor $q$ and offset $\left(\epsilon_{g}+0.5\right) \bmod 1$ are presented. We show here $\left(\epsilon_{g}+0.5\right)$ mod 1 as this is the full offset that relates to the position of the $g$-dominated modes, that is, the position of the "S-shape" in the period-échelle diagram. For the RGB stars $(\Delta \Pi<100 \mathrm{~s}$, green histograms $)$ we find $q$ values below 0.25 consistent with earlier results (Mosser et al. 2012b, $2017)$. For $\left(\epsilon_{g}+0.5\right)$ mod 1 in RGB stars we find values between roughly 0.3 and 1. A discussion on this is presented in Sect. 5.

In Fig. 5, we compare our derived values for the coupling factor with the values obtained by Mosser et al. (2017) and values from Corsaro (priv. comm.) for the stars that we have in common. Generally, the values are consistent within their uncertainties (see top panel of Fig. 5). However, we note that for RGB stars $(q<0.25)$, we find a linear correlation between the differences in $q$ (our values - literature) versus $q$ with a Pearson $r$ coefficient of 0.7 (bottom panel of Fig. 5). We also computed the t-statistic and use a two-sided $t$-test to find that we can reject a relation with zero slope at $>99 \%$ level. This correlation could be related to the fact that in our analysis we have left $\epsilon_{g}$ as a free parameter, while $\epsilon_{g}$ was kept fixed in the analyses already present in the literature.

\subsection{Core helium burning stars}

In this work we analyse the models by Constantino et al. (2015) as described in Table 1. The results of these models are mostly summarised in Table 1. From these results we conclude that our method can be applied to $\mathrm{CHeB}$ stars with non-spiky behaviour, where some irregular behaviour can be accounted for. For $\mathrm{CHeB}$ 

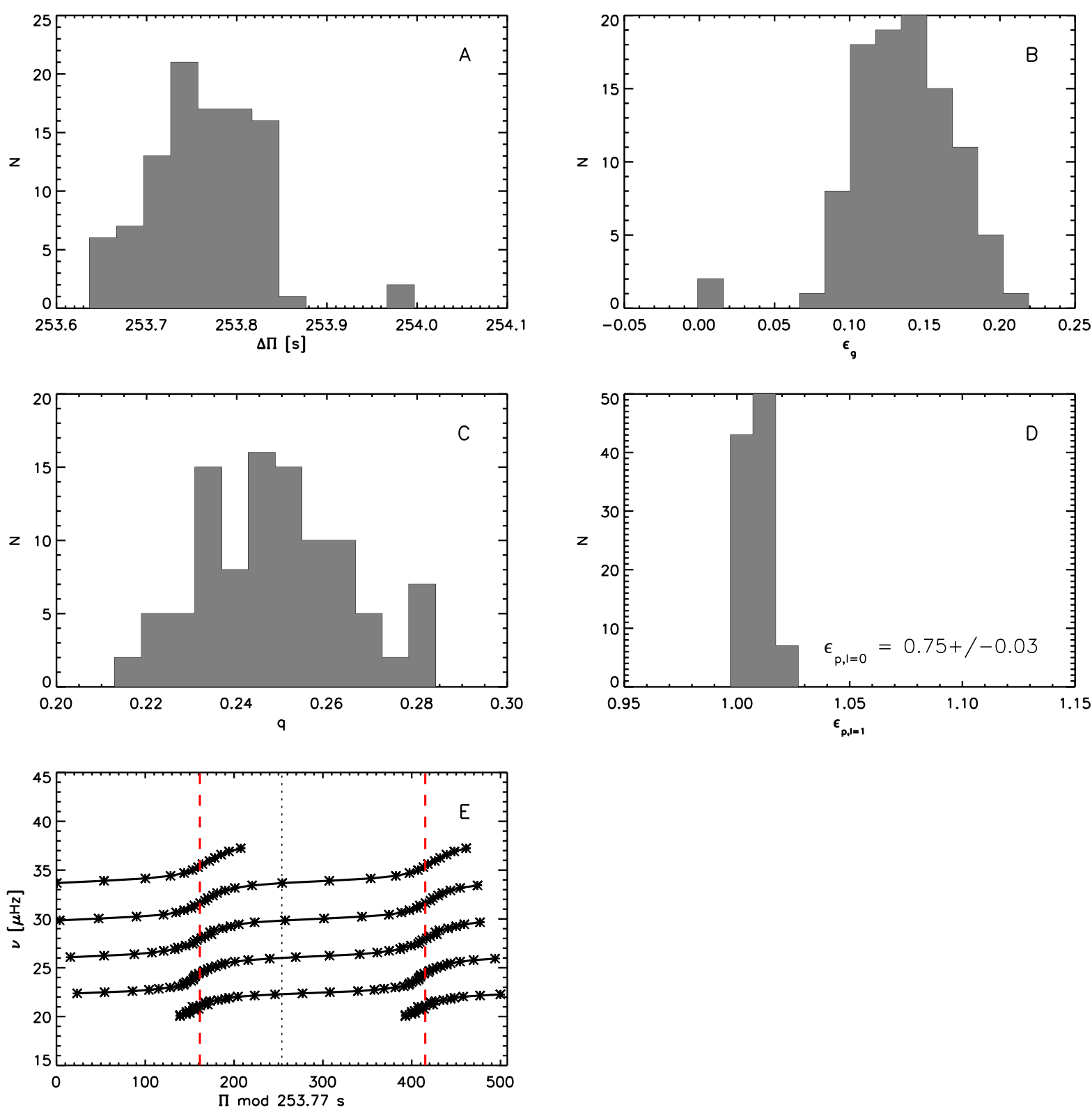

Fig. 2. As in Fig. 1 but for $\mathrm{CHeB}$ Model 7 with all frequencies selected in a $5 \Delta v$-wide range.

Model 2 we seem to be at the limit of the amount of irregularity the method can handle, although in some mode sets we do find the correct solution. For $\mathrm{CHeB}$ Model 9, we find, in addition to the irregularity, spikes in the mode inertia profile that most likely hamper the determination of the period spacing. For the analysis in the remainder of this paper we take $\mathrm{CHeB}$ Models 0 , $1,3,4,5,6,7,8,11$, and 14 (indicated with a $*$ in Table 1 ) into account. The results for these models are also shown in Fig. 3.

In terms of observations of $\mathrm{CHeB}$ stars we have frequencies for KIC 5000307, which is a lithium-rich star (Silva Aguirre et al. 2014). The current result for $\Delta \Pi$ is close ( $\sim 1 \%$ difference) to the value obtained by Silva Aguirre et al. (2014). Such a value is consistent with the results by Mosser et al. (2014) for a 1.5-2 $M_{\odot} \mathrm{CHeB}$ star.

For $\mathrm{CHeB}$ stars with $\Delta \Pi$ values that are deemed reliable, Fig. 4 shows the coupling factor $q$ and offset $\epsilon_{g}$. For the CHeB stars $(\Delta \Pi>200 \mathrm{~s}$, blue dashed histograms), we find $q$ values between 0.2 and 0.4 in agreement with earlier results (Mosser et al. 2012b, 2017).

\section{Discussion}

We have shown that we can constrain $\Delta \Pi, q, \epsilon_{g}$ and $\epsilon_{\mathrm{p} 1}$ for stars with enough observed dipole modes using the formalism proposed by Christensen-Dalsgaard (2012), Jiang \& Christensen-Dalsgaard (2014), Cunha et al. (2015), and Hekker $\&$ Christensen-Dalsgaard (2017). Based on these results, we first discuss what the obtained parameters reveal about the internal structures of the stars and subsequently investigate the impact of different mode sets.

\subsection{Period spacing $\Delta \Pi$}

For the models, we have compared the value of $\Delta \Pi$ obtained from frequencies using the approach outlined here and the 
Table 2. Results for the RGB stars and models.

\begin{tabular}{|c|c|c|c|c|c|c|c|}
\hline Star & $\Delta \Pi_{\text {lit }}[\mathrm{s}]$ & $\Delta \Pi[\mathrm{s}]$ & $\epsilon_{g}$ & $q$ & $\epsilon_{\mathrm{p} 1}$ & $\Delta v[\mu \mathrm{Hz}]$ & $p$ \\
\hline KIC003744043 & 75.98 & $76.05_{0.10}^{0.09}$ & $0.9_{0.1}^{0.1}$ & $0.140_{0.010}^{0.010}$ & $0.810_{0.047}^{0.010}$ & $9.841 \pm 0.006$ & 1.00 \\
\hline $\mathrm{KIC} 004448777^{b}$ & 89.87 & $89.33_{0.03}^{0.02}$ & $0.29_{0.01}^{0.01}$ & $0.155_{0.001}^{0.002}$ & $0.8841_{0.0007}^{0.0006}$ & $16.921 \pm 0.006$ & 0.66 \\
\hline $\mathrm{KIC} 005866737^{a}$ & 68.49 & $66.014_{0.006}^{0.005}$ & $0.008_{0.008}^{0.012}$ & $0.093_{0.005}^{0.006}$ & $0.686_{0.003}^{0.003}$ & $6.499 \pm 0.006$ & 0.42 \\
\hline KIC006117517 & 76.91 & $76.86_{0.06}^{0.06}$ & $0.09_{0.08}^{0.10}$ & $0.15_{0.03}^{0.02}$ & $0.872_{0.005}^{0.005}$ & $10.031 \pm 0.006$ & 1.00 \\
\hline KIC006144777 & 79.23 & $79.04_{0.04}^{0.02}$ & $0.24_{0.03}^{0.04}$ & $0.121_{0.007}^{0.007}$ & $0.80_{0.01}^{0.01}$ & $10.956 \pm 0.004$ & 1.00 \\
\hline KIC007060732 & 77.10 & $77.76_{0.04}^{0.05}$ & $0.14_{0.06}^{0.05}$ & $0.129_{0.008}^{0.006}$ & $0.799_{0.004}^{0.003}$ & $10.853 \pm 0.004$ & 0.81 \\
\hline KIC007619745 & 79.17 & $79.04_{0.09}^{0.06}$ & $0.13_{0.05}^{0.09}$ & $0.144_{0.011}^{0.009}$ & $0.869_{0.051}^{0.006}$ & $13.059 \pm 0.006$ & 0.95 \\
\hline 08366230 & & $87.84_{0.09}^{0.15}$ & $0.25_{0.10}^{0.07}$ & $0.14_{0.01}^{0.01}$ & $0.866_{0.004}^{0.005}$ & $13.619 \pm 0.006$ & 0.97 \\
\hline KIC008475025 & 74.80 & $74.46_{0.06}^{0.03}$ & $1.00_{0.05}^{0.09}$ & $0.126_{0.007}^{0.009}$ & $0.764_{0.003}^{0.004}$ & $9.572 \pm 0.004$ & 1.00 \\
\hline KIC008718745 & & $79.99_{0.03}^{0.03}$ & $0.34_{0.04}^{0.05}$ & $0.146_{0.006}^{0.008}$ & $0.788_{0.003}^{0.003}$ & $11.363 \pm 0.005$ & 0.98 \\
\hline KIC0091459 & 76.98 & $77.023_{0.008}^{0.008}$ & $0.983_{0.011}^{0.010}$ & $0.155_{0.002}^{0.002}$ & $0.8800_{0.0009}^{0.0009}$ & $10.882 \pm 0.005$ & 1.00 \\
\hline KIC009145955 & 77.01 & $76.78_{0.05}^{0.06}$ & $0.31_{0.06}^{0.08}$ & $0.17_{0.02}^{0.01}$ & $0.827_{0.005}^{0.007}$ & $10.941 \pm 0.005$ & 1.00 \\
\hline KIC009267654 & 78.41 & $78.13_{0.09}^{0.09}$ & $0.93_{0.10}^{0.13}$ & $0.14_{0.01}^{0.01}$ & $0.798_{0.007}^{0.007}$ & $10.239 \pm 0.004$ & 0.79 \\
\hline KIC009475697 & 75.70 & $75.54_{0.05}^{0.04}$ & $0.23_{0.07}^{0.09}$ & $0.18_{0.02}^{0.02}$ & $0.797_{0.005}^{0.005}$ & $9.806 \pm 0.004$ & 0.56 \\
\hline KIC009882316 & 80.59 & $80.42_{0.10}^{0.08}$ & $0.14_{0.07}^{0.09}$ & $0.19_{0.01}^{0.01}$ & $0.860_{0.007}^{0.008}$ & $13.602 \pm 0.007$ & 1.00 \\
\hline KIC010123207 & 83.88 & $83.59_{0.06}^{0.06}$ & $0.28_{0.06}^{0.05}$ & $0.18_{0.01}^{0.01}$ & $0.836_{0.006}^{0.004}$ & $13.629 \pm 0.007$ & 1.00 \\
\hline $\mathrm{KIC} 010200377^{a}$ & 81.54 & $81.300_{0.009}^{0.010}$ & $0.338_{0.011}^{0.009}$ & $0.155_{0.002}^{0.002}$ & $0.7820_{0.0008}^{0.0006}$ & $12.501 \pm 0.007$ & 1.00 \\
\hline KIC010200377 & 81.58 & $81.46_{0.03}^{0.04}$ & $0.15_{0.05}^{0.04}$ & $0.19_{0.01}^{0.02}$ & $0.895_{0.005}^{0.005}$ & $12.377 \pm 0.004$ & 1.00 \\
\hline KIC010257278 & 79.81 & $79.72_{0.05}^{0.07}$ & $0.08_{0.05}^{0.07}$ & $0.143_{0.009}^{0.009}$ & $0.81_{0.01}^{0.06}$ & $12.114 \pm 0.005$ & 0.89 \\
\hline KIC011353313 & 76.00 & $77.15_{0.12}^{0.09}$ & $0.0_{0.1}^{0.2}$ & $0.15_{0.02}^{0.01}$ & $0.772_{0.010}^{0.017}$ & $10.724 \pm 0.006$ & 1.00 \\
\hline KIC011913545 & 77.84 & $77.79_{0.06}^{0.09}$ & $0.08_{0.08}^{0.07}$ & $0.123_{0.008}^{0.014}$ & $0.790_{0.002}^{0.036}$ & $10.092 \pm 0.004$ & 0.98 \\
\hline KIC011968334 & 78.10 & $77.79_{0.05}^{0.07}$ & $0.45_{0.09}^{0.07}$ & $0.13_{0.01}^{0.01}$ & $0.822_{0.004}^{0.004}$ & $11.363 \pm 0.005$ & 1.00 \\
\hline KIC012008916 & 80.47 & $81.4_{0.2}^{0.3}$ & $0.2_{0.2}^{0.2}$ & $0.09_{0.02}^{0.01}$ & $0.804_{0.008}^{0.044}$ & $12.834 \pm 0.005$ & 0.62 \\
\hline RGBmodel 0 & 82.61 & $82.239_{0.008}^{0.007}$ & $0.317_{0.009}^{0.009}$ & $0.141_{0.002}^{0.002}$ & $0.5212_{0.0008}^{0.0008}$ & $11.977 \pm 0.003$ & 1.00 \\
\hline RGBmodel 1 & 73.49 & $73.216_{0.006}^{0.007}$ & $0.30_{0.02}^{0.01}$ & $0.126_{0.004}^{0.005}$ & $0.501_{0.002}^{0.002}$ & $7.136 \pm 0.003$ & 1.00 \\
\hline RGBmodel 2 & 62.15 & $62.036_{0.006}^{0.005}$ & $0.14_{0.04}^{0.05}$ & $\begin{array}{r}0.079_{0.008}^{0.009} \\
\end{array}$ & $1.357_{0.004}^{0.005}$ & $4.186 \pm 0.003$ & 0.55 \\
\hline
\end{tabular}

Notes. For most stars the results are based on the frequencies presented by Corsaro et al. (2015). Stars indicated with superscript (a) show results based on frequencies presented by Datta et al. (2015) and the star indicated with superscript $(b)$ shows results based on frequencies presented by Di Mauro et al. (2016). For the models we show results for the mode set with all dipole frequencies included in a $5 \Delta v$-wide range.

asymptotic value $\left(\Delta \Pi_{l, \text { asymptotic }}\right)$ computed through the integral of the Brunt-Väisälä frequency:

$\Delta \Pi_{l, \text { asymptotic }}=\frac{2 \pi^{2}}{l(l+1)}\left(\int_{r_{1}}^{r_{2}} N \frac{\mathrm{d} r}{r}\right)^{-1}$,

with $N$ being the Brunt-Väisälä frequency and $r_{1}$ and $r_{2}$ being the lower and upper turning points ${ }^{3}$.

\footnotetext{
3 In practice we computed one value for $\Delta \Pi_{l, \text { asymptotic }}$ per model taking the integral over the total area of the Brunt-Väisälä frequency and not a separate value for each frequency with its specific turning points. The difference in the values is however negligible compared to the differences we discuss here.
}

The observed and computed asymptotic values are in broad agreement; for RGB models the values computed from the Brunt-Väisälä frequency are slightly higher (typically of the order 0.01 to a few times $0.1 \mathrm{~s}$ ) than the one obtained from the frequencies. This is as expected from theory and in line with earlier results (e.g. Datta et al. 2015). For the CHeB models we find that our $\Delta \Pi$ values obtained from frequencies are larger than the asymptotic reference values (up to about $10 \mathrm{~s}$, see Fig. 3). These relatively high values for $\Delta \Pi$ seem to be consistent with earlier findings by, for example, Mosser et al. (2014) and studied in more detail by Constantino et al. (2015). These authors report systematic differences in $\Delta \Pi$ of $\mathrm{CHeB}$ stars between observations (using frequencies) and model predictions (using the Brunt-Väisälä frequency). The larger values of $\Delta \Pi$ obtained 
Table 3. As in Table 2 for $\mathrm{CHeB}$ stars.

\begin{tabular}{lccccccc}
\hline \hline Star & $\Delta \Pi_{\text {lit }}[\mathrm{s}]$ & $\Delta \Pi[\mathrm{s}]$ & $\epsilon_{g}$ & $q$ & $\epsilon_{\mathrm{p} 1}$ & $\Delta v[\mu \mathrm{Hz}]$ & $p$ \\
\hline KIC005000307 & 319.95 & $322.2_{0.1}^{0.1}$ & $0.48_{0.02}^{0.03}$ & $0.34_{0.02}^{0.01}$ & $0.628_{0.004}^{0.005}$ & $4.724 \pm 0.002$ & 0.65 \\
\hline CHeBmodel 0 & 240.00 & $241.98_{0.02}^{0.01}$ & $0.989_{0.006}^{0.006}$ & $0.24_{0.02}^{0.02}$ & $1.051_{0.004}^{0.006}$ & $3.527 \pm 0.003$ & 0.86 \\
\hline CHeBmodel 1 & 238.00 & $243.32_{0.07}^{0.06}$ & $0.36_{0.03}^{0.05}$ & $0.22_{0.01}^{0.02}$ & $1.049_{0.006}^{0.004}$ & $3.584 \pm 0.003$ & 0.99 \\
\hline CHeBmodel 3 & 247.00 & $249.83_{0.07}^{0.04}$ & $0.57_{0.03}^{0.04}$ & $0.23_{0.01}^{0.02}$ & $0.941_{0.005}^{0.006}$ & $3.611 \pm 0.003$ & 1.00 \\
\hline CHeBmodel 4 & 252.00 & $253.30_{0.05}^{0.04}$ & $0.15_{0.03}^{0.03}$ & $0.249_{0.014}^{0.009}$ & $1.004_{0.004}^{0.006}$ & $3.734 \pm 0.003$ & 0.68 \\
\hline CHeBmodel 5 & 253.00 & $253.80_{0.04}^{0.06}$ & $0.31_{0.03}^{0.02}$ & $0.26_{0.01}^{0.02}$ & $1.400_{0.005}^{0.004}$ & $3.908 \pm 0.003$ & 1.00 \\
\hline CHeBmodel 6 & 253.00 & $253.82_{0.04}^{0.06}$ & $0.33_{0.03}^{0.02}$ & $0.26_{0.01}^{0.02}$ & $1.400_{0.005}^{0.006}$ & $3.908 \pm 0.003$ & 1.00 \\
\hline CHeBmodel 7 & 253.00 & $253.76_{0.06}^{0.06}$ & $0.14_{0.03}^{0.03}$ & $0.25_{0.02}^{0.02}$ & $1.008_{0.005}^{0.006}$ & $3.740 \pm 0.003$ & 0.98 \\
\hline CHeBmodel 8 & 314.00 & $315.96_{0.08}^{0.07}$ & $0.29_{0.02}^{0.03}$ & $0.29_{0.01}^{0.02}$ & $1.349_{0.004}^{0.006}$ & $3.810 \pm 0.003$ & 1.00 \\
\hline CHeBmodel 11 & 273.00 & $274.09_{0.06}^{0.06}$ & $0.43_{0.03}^{0.02}$ & $0.28_{0.01}^{0.02}$ & $1.334_{0.004}^{0.004}$ & $4.090 \pm 0.003$ & 1.00 \\
\hline CHeBmodel 14 & 268.00 & $277.30_{0.07}^{0.06}$ & $0.49_{0.02}^{0.03}$ & $0.28_{0.02}^{0.01}$ & $1.325_{0.006}^{0.004}$ & $3.743 \pm 0.003$ & 1.00 \\
\hline
\end{tabular}

from frequencies may indicate that in $\mathrm{CHeB}$ stars the frequencies are not sensitive to the whole buoyancy cavity, possibly due to a discontinuity, or additional convective areas blocking the oscillations. Bossini et al. (2017) indeed find that additional mixing in terms of core-overshooting can mitigate the differences in $\Delta \Pi$ between observations and models.

\subsection{Coupling term $q$}

It is known that $q$ provides information about the coupling of the wave in the gravity and acoustic cavity, with $q=0$ for no coupling and $q=1$ for full coupling (e.g. Takata 2016b). In the current study, we would like to further our understanding of the dependence of $q$ on physical parameters of the star. Following Takata (2016b) and Mosser et al. (2017) it is possible to compute

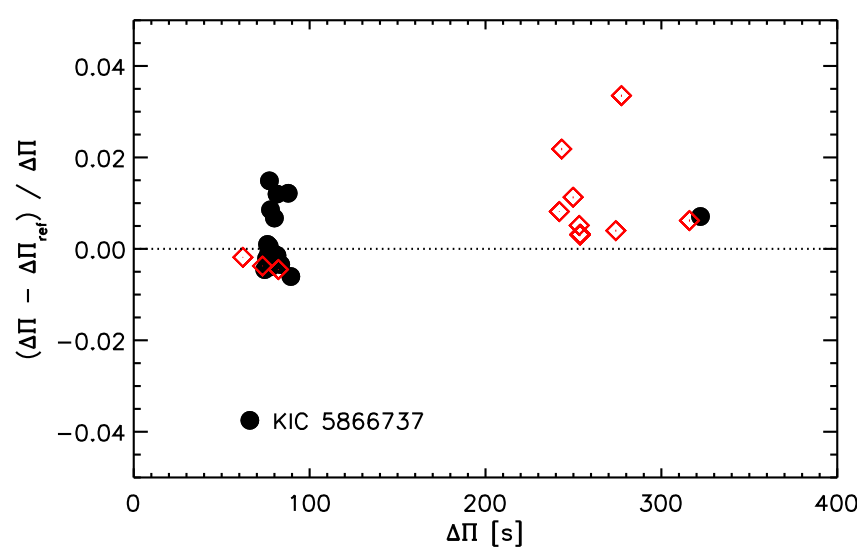

Fig. 3. Comparison of the period spacings derived in this work with reference values, where, for the models, the reference values are computed from the integral of the Brunt-Väisälä frequency (Eq. (8)), and for real data, reference values are observed values from the literature with updated values for KIC 6144777 and KIC 7060732; see text for details. Results for real stars and models are shown with black dots and open red diamonds, respectively. Error bars are mostly smaller than the symbol size. Precise agreement is highlighted by the dotted line. $q$ for the models for each frequency using the following general formulation:

$q=\frac{1-\sqrt{1-T^{2}}}{1+\sqrt{1-T^{2}}}$,

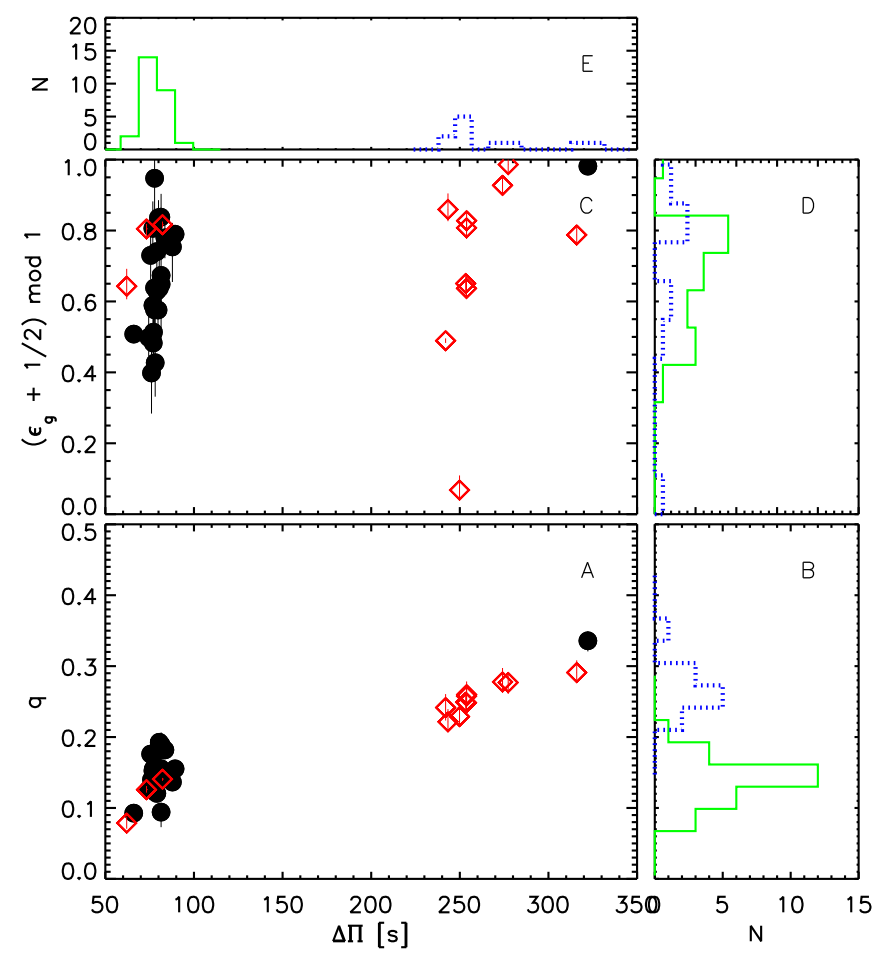

Fig. 4. Panel $A$ : coupling factor $q$ vs. $\Delta \Pi$ for all objects. Real stars are indicated with black dots and models with red diamonds. Panel $B$ : histogram of $q$ for RGB stars (real stars + models) and CHeB stars in green solid and blue dotted lines, respectively. Panel $C$ : as in panel $A$, now for $\left(\left(\epsilon_{g}+1 / 2\right) \bmod 1\right)$ vs. $\Delta \Pi$. Panel $D$ : distribution of $\left(\epsilon_{g}+1 / 2\right) \bmod 1$. Panel $E$ : the distribution of $\Delta \Pi$ results. The colour-coding and line styles in panels $D$ and $E$ are the same as in panel $B$. In panels $A$ and $C$, uncertainties are over plotted. These are, however, in a number of cases smaller than the symbol size. 


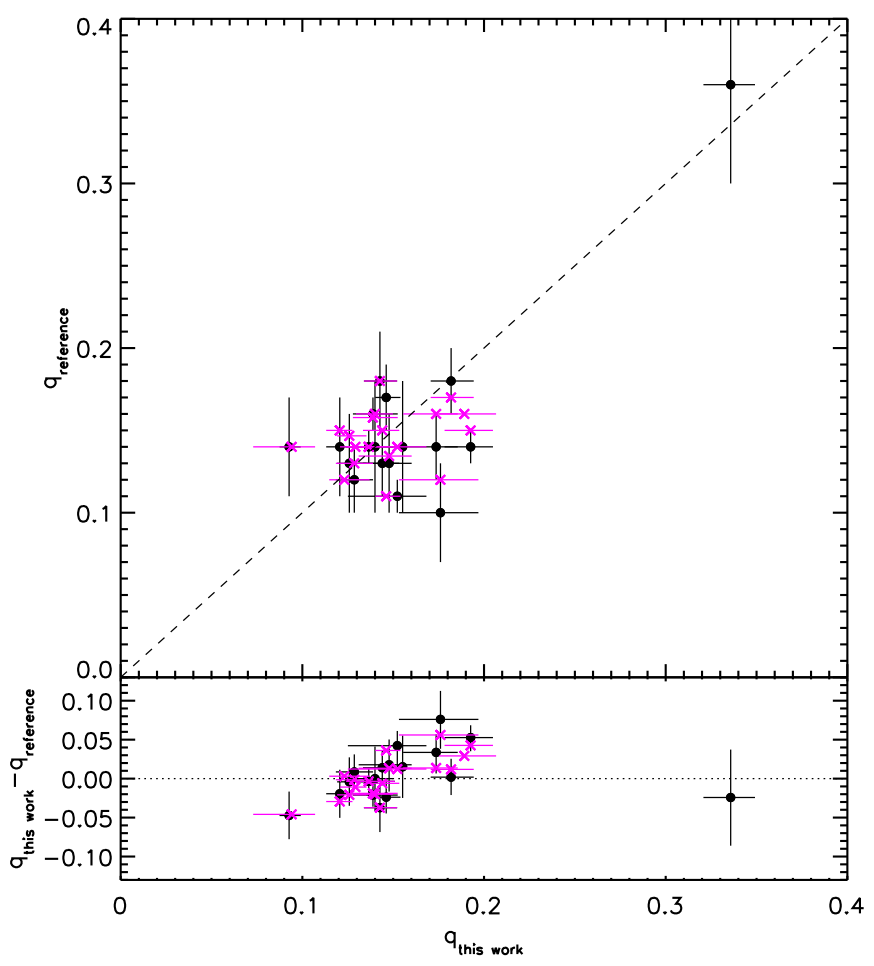

Fig. 5. Top: comparison between the coupling factor $q_{\text {this work }}$ determined in this work and the values $q_{\text {reference }}$ obtained by Mosser et al. (2017, black dots) and Corsaro (priv. comm., magenta crosses) for the stars that we have in common. The dotted line indicates agreement. Bottom: difference between the coupling in this work and in the reference values in the sense $q_{\text {this work }}-q_{\text {reference }}$. We note that no uncertainties are provided by Corsaro.

with $T$ being the amplitude transmission coefficient, that is, a measure of how much of the wave passes through a barrier (in this case the evanescent zone), computed as

$T=\exp \left(-\int_{D} \kappa \mathrm{d} r\right)$

with $D$ being the radial extent of the evanescent region and $\kappa$ the radial wave vector:

$\kappa=\frac{\sqrt{\left(S_{1}^{2}-\omega^{2}\right)\left(\omega^{2}-N^{2}\right)}}{c \omega}$,

with $S_{1}$ being the Lamb frequency of a dipole mode, $N$ the Brunt-Väisälä frequency, $\omega$ the angular frequency equal to $2 \pi v$ and $c$ the sound speed.

For the computed values of $q$ we find a different value for each oscillation mode due to the fact that each oscillation mode has a different $\kappa$ (Eq. (11)) and encounters a slightly different evanescent zone as a consequence of the shape of the BruntVäisälä and Lamb frequencies. However, when extracting $q$ from observations, only one global value can be obtained as only the ensemble of dipole modes contains sufficient information to extract $q$. A comparison of the results of the computed $q$ values of the models with the value of $q$ obtained from the analysis using the method described in this paper is shown in Fig. 6. The results are generally consistent for $q>0.1$, while for weaker coupling we find that $q_{\text {observed }}$ is over-estimated compared to the values obtained from the models. This is consistent with the results by Mosser et al. (2017), who find that the computed

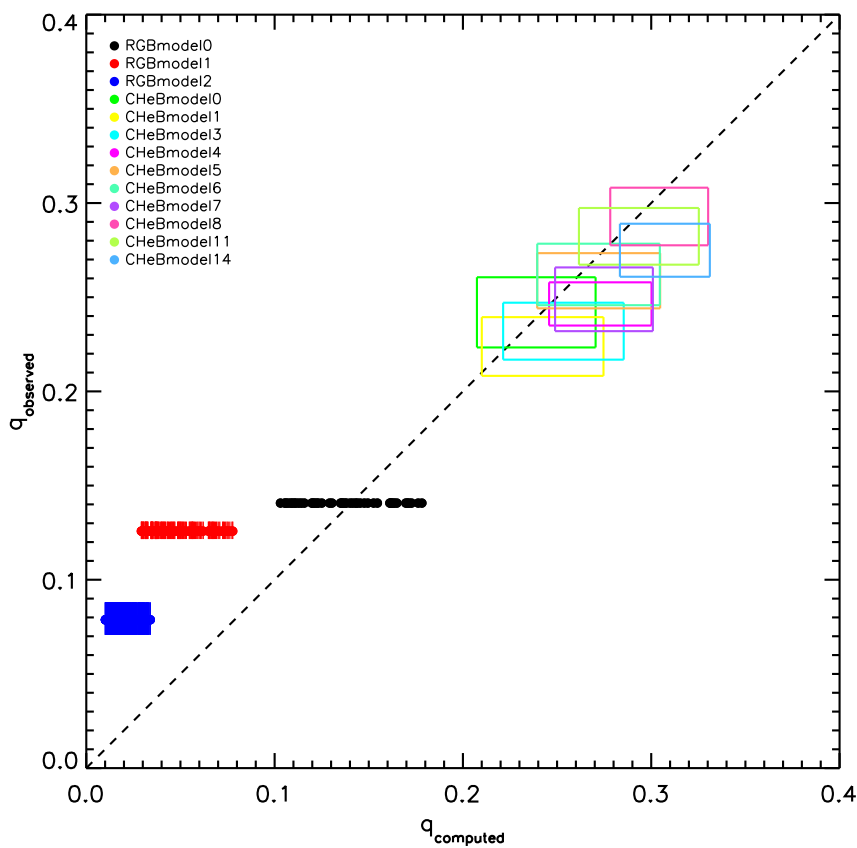

Fig. 6. Coupling factor computed using the method outlined in Sect. 2 $\left(q_{\text {observed }}\right)$ vs. the coupling factor for each frequency computed using Eqs. (9)-(11) $\left(q_{\text {computed }}\right)$. For visual purposes we show the individual points with uncertainties for the RGB models, and a rectangle for the $\mathrm{CHeB}$ models that comprises the results including the uncertainties for that particular model; the horizontal width of each rectangular box indicates the spread in $q$ computed through Eqs. (9)-(11) for all frequencies in a range of five times $\Delta v$ centred around $v_{\max }$. The legends show the colour with which each model is indicated.

coupling factor of a model high on the RGB has a significantly smaller value than that observed; the reason for this remains unclear.

We subsequently investigate the dependence of $q$ on physical parameters of the star and find empirically that $q$ shows a tight correlation with $\Delta r / r_{\text {evanescent }}$, which is the radial extent of the evanescent zone normalised by the radius of the (midpoint of the) evanescent zone $r_{\text {evanescent }}$. This correlation extends over

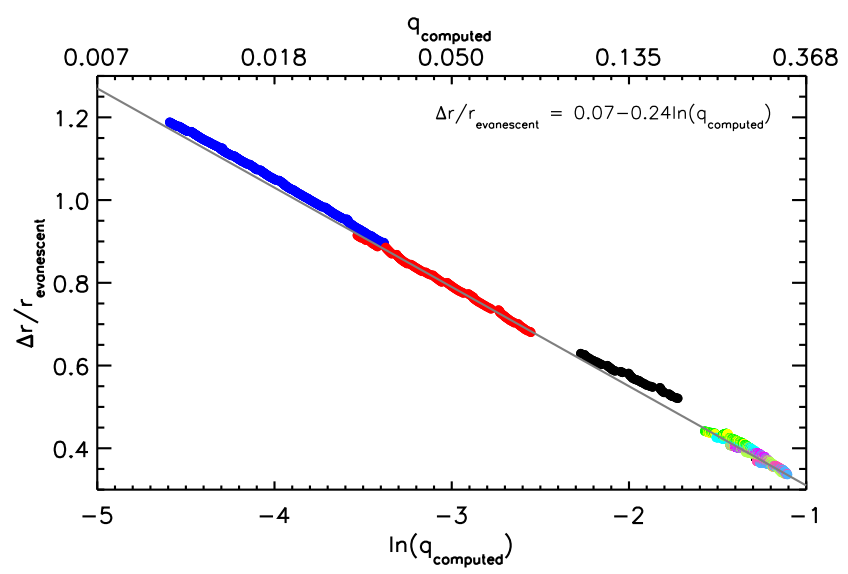

Fig. 7. Radial extent of the evanescent zone $(\Delta r)$ normalised by the radius of the midpoint of the evanescent zone $\left(r_{\text {evanescent }}\right)$ vs. the natural logarithm of the coupling factor $\left(\ln \left(q_{\text {computed }}\right)\right)$ for each frequency in the frequency range $v_{\max } \pm 3.5 \Delta v$ computed using Eqs. (9)-(11). The colour-coding is the same as in Fig. 6 . The grey line indicates the fit as indicated in the legend (see text for more details). The values of $q_{\text {computed }}$ are shown in the top axis. 
the full range of $q$ covered by our models except for a small deviation for RGB Model 0 at its highest $q$ values (see Fig. 7). We use symbolic regression to find a functional fit. With this symbolic regression we find that $\Delta r / r_{\text {evanescent }}$ is linearly related to $\ln \left(q_{\text {computed }}\right)$, where $q_{\text {computed }}$ is computed using Eqs. (9)-(11):

$\Delta r / r_{\text {evanescent }}=0.07-0.24 \ln \left(q_{\text {computed }}\right)$.

This linear behaviour is expected theoretically (see Eqs. (9)-(11)) and vindicated by the use of the symbolic regression. We speculate that RGB Model 0 deviates from the relation because this model is the least evolved compared to the other models and still on its way to the homology that is apparent in the other models. We note that the coefficients in Eq. (12) are valid for $1 M_{\odot}$ models with solar metallicity and may differ depending on mass and metallicity.

\subsection{Offset $\epsilon_{g}$}

We now consider $\epsilon_{g}$. In this work we have kept $\epsilon_{g}$ a free parameter and checked whether $\left(\epsilon_{g}+0.5\right) \bmod 1$ is consistent with the position of the g-dominated mode in the period-échelle diagram (red dashed lines in panel E of Fig. 1). This is indeed the case. We use a Kolmogorov-Smirnov test to verify that the distribution of $\epsilon_{g}$ that we find is consistent with the distribution of $\epsilon_{g}$ fixed to 0 . For the observational data (black dots in Fig. 4) the probability of the cumulative distributions being the same is $2.6 \times 10^{-8}$. For the models, the probability of the cumulative distributions being the same is $5.3 \times 10^{-8}$. In the case of the combined sample, the consistency is vanishingly small with a probability of $1.8 \times 10^{-12}$. As a final check, we performed the analysis as described in Sect. 2 with $\epsilon_{g}$ fixed at 0 . In a number of these cases $\left(\epsilon_{g}+0.5\right) \bmod 1$ is not consistent with the location of the $g$-dominated modes in the period-échelle diagram. Hence, we conclude that $\epsilon_{g}$ should not be kept fixed.

It is noteworthy that for RGB Models 1 and 2, the value of $q$ decreased towards the theoretical value when keeping $\epsilon_{g}$ fixed. As the period-échelle diagram did not reflect the value of $\epsilon_{g}$ we do not trust these results. It does however indicate a correlation between $\epsilon_{g}$ and $q$. Indeed, for the stars and models considered here we find a linear correlation between $q$ and $\epsilon_{q}$ with a Pearson $\mathrm{r}$ coefficient of 0.34 . We note that this correlation may be (partly) responsible for the correlation evident in Fig. 5.

The results in Fig. 4 show that there is a preference for $\epsilon_{g}$ to be larger than 0.3 for RGB stars. Our sample of stars is too small to judge whether this is a coincidence, whether this is due to selection effects, or whether this is real.

\subsection{Offset $\epsilon_{\mathrm{p} 1}$}

As discussed previously, the frequencies at which the radial and dipole pressure modes are observed are described with the help of two offsets, $\epsilon_{\mathrm{p} 0}$ for the radial modes and $\epsilon_{\mathrm{p} 1}$ for the dipole modes. Here we investigate the assumption that $\epsilon_{p l}=\epsilon_{p 0}+l / 2$ (Sect. 2.1). We show in Fig. 8 that indeed most of the $\left(\epsilon_{\mathrm{p} 0}+0.5\right)-\epsilon_{\mathrm{p} 1}$ values (i.e. equivalent with $\left.\delta v_{01} / \Delta v\right)$ for the RGB stars cluster around zero. We note a decrease of $\left(\epsilon_{\mathrm{p} 0}+0.5\right)-\epsilon_{\mathrm{p} 1}$ for $\mathrm{CHeB}$ models towards lower values of $\Delta v$ similar to what was presented by Corsaro et al. (2012) for $\delta v_{01} / \Delta v$ for $\mathrm{CHeB}$ stars. The main discrepancies lie in the five models that have values for $\left(\epsilon_{\mathrm{p} 0}+0.5\right)-\epsilon_{\mathrm{p} 1}$ above 0.1 . These are $\mathrm{CHeB}$ models $0,1,3,4$ and 7. For these models we checked the frequencies and the values for $\epsilon_{\mathrm{p} 0}$ and $\epsilon_{\mathrm{p} 1}$ obtained by our method do reflect the locations of the radial modes and $p$ dominated dipole modes, respectively. Therefore, it may be that

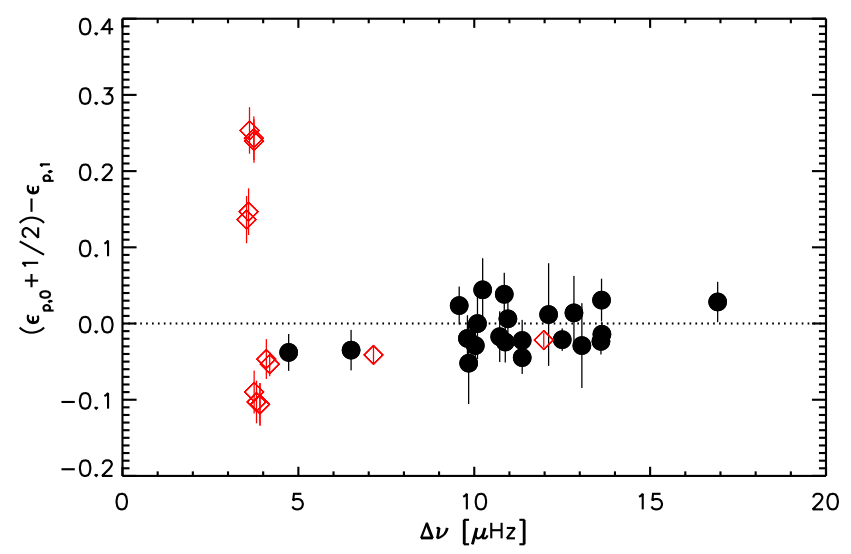

Fig. 8. Values of $\left(\epsilon_{\mathrm{p} 0}+0.5\right)-\epsilon_{\mathrm{p} 1}$ vs. $\Delta v$ for observed stars (black dots) and models (red diamonds).

this is due to the structure of the model. We find that the models that have positive values of $\left(\epsilon_{\mathrm{p} 0}+0.5\right)-\epsilon_{\mathrm{p} 1}$ are all the original models or have mode inertias that have not shifted compared to the original models.

\subsection{Cavity boundaries}

Recently, Mosser et al. (2017) suggested that the local density contrasts of the core $\beta_{N}=-\frac{\mathrm{d} \ln N}{\mathrm{~d} \ln r}$ and the envelope $\beta_{S}=-\frac{\mathrm{d} \ln S_{1}}{\mathrm{~d} \ln r}$ are approximately equal:

$$
-\frac{\mathrm{d} \ln N}{\mathrm{~d} \ln r}=\beta_{N} \simeq \beta \simeq \beta_{S}=-\frac{\mathrm{d} \ln S_{1}}{\mathrm{~d} \ln r} \text {. }
$$

This suggestion is based on the fact that the Brunt-Väisälä frequency and the Lamb frequency show similar radial variations for the frequencies probed in the region between the hydrogenburning shell and the base of the convective envelope where the evanescent zone is located. This assumption is based on the analysis by Takata (2016b), which is inspired by models with $\Delta v>20 \mu \mathrm{Hz}$. For our analysis of more evolved RGB stars (with $\Delta v<15 \mu \mathrm{Hz}$ ), the propagation diagram (Fig. 9) shows that this seems a valid approximation for $\mathrm{CHeB}$ stars, but not for the RGB stars analysed in this work that are more evolved than the ones addressed by Takata (2016b).

Further investigation of $\beta_{N}$ shows that these values increase with decreasing values of frequency for RGB stars (Fig. 10). In this figure we have removed oscillation modes for which the computation of $\kappa$ (Eq. (11)) was hampered by the spike in the Brunt-Väisälä frequency due to the discontinuity in the mean molecular weight at the deepest extent of the convection zone. We note that $\beta_{N} \cong 1$ for $\mathrm{CHeB}$ stars and does not show significant variation with frequency.

Takata (2016a,b), and references therein, show that $\epsilon_{g}$ depends on the phase lags introduced at both the inner and outer turning points of the wave as well as the reflection coefficient of the edges. Figure 10 indicates that $\beta_{N}$ shows trends with $v$ (and with $q$ ). We expect that this could induce a different reflection coefficient and hence could show a correlation with $\epsilon_{g}$. We indeed see a decrease in $\epsilon_{g}$ with the evolution along the RGB (that is with models with decreasing frequencies) indicated with the diamonds and the right axis in the left panel of Fig. 10. This could indicate that a larger density contrast at the edge of the $g$-mode cavity would cause a lower offset $\epsilon_{g}$. More models are required to confirm this trend.

For $\beta_{S}$, Takata (2016a) predict an upper limit for RGB stars of 1.5. We find consistent results for our models with a roughly 


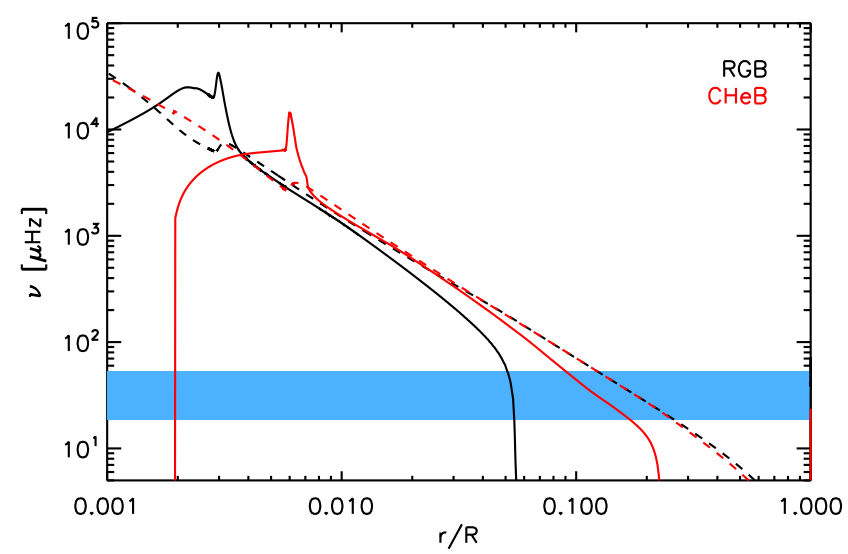

Fig. 9. Propagation diagram of a RGB (black) and $\mathrm{CHeB}$ model (red) with the Brunt-Väisälä frequencies indicated by the solid lines and the Lamb frequencies by the dashed lines. The region in which we can expect oscillations to be observed is indicated with the blue bar.

constant value of $\beta_{S} \cong 1.35$, with an increasing value towards lower frequency.

\subsection{Impact of different mode sets and correlations}

In some cases, different mode sets arise from independent analysis of the stars, and in other cases we choose different mode sets from stellar models using different criteria to select the modes.

For the different mode sets of the stars KIC 9145955 and KIC 10200377 we see substantial overlap in the detected modes and their frequencies (see Fig. A.1), which results in derived values for $\Delta \Pi, q, \epsilon_{g}, \epsilon_{\mathrm{p} 1}$, and $\Delta v$ that differ outside the quoted uncertainties. As $\Delta v$ is obtained from the radial modes and is kept fixed during the remainder of the procedure, the difference in this parameter may have an impact on the other results; we see that the results are frequency dependent and that formal uncertainties as quoted here do not account for that.

Furthermore, for both the models and the observed stars we find clear correlations between the obtained $\Delta \Pi$ and $\epsilon_{g}$. All different mode sets of a particular model show a trend in which $\left(\epsilon_{g}+0.5\right)$ mod 1 decreases with increasing $\Delta \Pi$, as expected

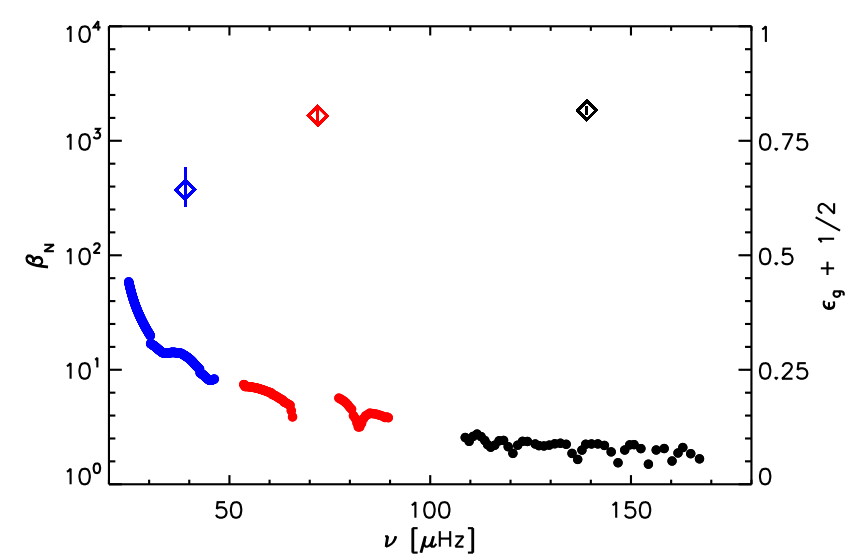

Fig. 10. Local density contrast of the core $\beta_{N}$ vs. frequency for the three RGB models. The diamonds indicate the value of $\epsilon_{g}$ obtained for each model, considering all dipole frequencies in a $5 \Delta v$-wide frequency range, as per the right-hand axis. The colour-coding is the same as in Fig. 6. from Eq. (2). For the $\mathrm{CHeB}$ models, we additionally see that, firstly, the wider frequency range always leads to lower values of $\left(\epsilon_{g}+0.5\right) \bmod 1$ and a higher value of $\Delta \Pi$ and, secondly, that this is the case irrespective of the selections of the frequencies in this range (at least for the two sets investigated here). For the RGB models, the correlation with the different datasets with different frequency ranges is not so clear.

The trends that are present in our results are similar to the ones between $\Delta v$ and $\epsilon_{p}$ for the acoustic modes. For the acoustic modes these differences are related to the fact that $\Delta v$ is a function of frequency due to stellar structure changes on long and short scales. From theory it is also expected that the period spacing is frequency dependent, which is what appears in our current results. This, together with the fact that we find systematic differences between $\Delta \Pi$ obtained from frequencies and from the integral of the Brunt-Väisälä frequency, is a direct indication that a comparison of $\Delta \Pi$, as well as other parameters, obtained from the same sets of frequencies in both observations and models is essential for detailed comparisons.

\section{Conclusions}

In this work we have investigated the use of the formalism by Jiang \& Christensen-Dalsgaard (2014) for RGB and CHeB stars in obtaining values for $\Delta \Pi, q, \epsilon_{g}$, and $\epsilon_{\mathrm{p} 1}$ from individual frequencies. This formalism provides a global solution based on all dipole modes with the same azimuthal order and can be applied to all azimuthal orders for which the results are combined in the current work.

The fact that the radial order is explicitly included in this formalism reduces problems with alias results that are present in other methods and provides the possibility to constrain $\epsilon_{g}$. On the other hand we find that for cases with weak coupling, for example, KIC 5866737, the lower number of frequencies and the higher radial order provide challenges to the method, resulting in reduced reliability of the results.

The current results indicate that for RGB stars $\left(\epsilon_{g}+0.5\right)$ mod 1 can be constrained and is in all cases analysed here between 0.3 and 1 . We furthermore show that the local density contrast at the edge of the $g$-mode cavity $\left(\beta_{N}\right)$ does follow a trend with $\epsilon_{g}$. However, this requires further analysis. Additionally, we find that there is systematic overestimation of $\Delta \Pi$ for $\mathrm{CHeB}$ stars when computed from frequencies compared to the asymptotic value computed from the integral of the Brunt-Väisälä frequency, as already mentioned in the literature. We subsequently indicate that $\Delta \Pi$ and $\left(\epsilon_{g}+0.5\right)$ mod 1 depend on the mode set from which they are determined, where, for $\mathrm{CHeB}$ models, mode sets covering a wider frequency range provide higher values for $\Delta \Pi$ and lower values for $\left(\epsilon_{g}+0.5\right) \bmod 1$. These trends are typically not included in the quoted uncertainties. To mitigate this when performing a model comparison we deem it vital to treat data and models in the same way for a meaningful result. Finally, the values for $\epsilon_{\mathrm{p} 1}$ for the $\mathrm{CHeB}$ models may indicate that additional physics must be included in the models as presented by Constantino et al. (2015).

From the models, we find a linear correlation between the relative width of the evanescent zone normalised by its location $\left(\Delta r / r_{\text {evanescent }}\right)$ and the natural logarithm of the coupling factor $q$.

To further explore these conclusions and investigate the differences and trends in more depth, larger sets of observed stars and systematically chosen models for which individual frequencies are available need to be investigated. Methods to extract 
individual frequencies from the power spectra of time-series data are being developed (e.g. Corsaro et al. 2015; Garcia Saravia Ortiz de Montellano et al., in prep.) and the frequencies of larger sets of observed stars are expected in the near future.

Acknowledgements. We thank Abishek Datta, Anwesh Mazumdar and Thomas Constantino for kindly providing us with the models for RGB stars (Datta et al. 2015) and $\mathrm{CHeB}$ stars (Constantino et al. 2015). Additionally, we thank the anonymous referee, Joergen Christensen-Dalsgaard, Enrico Corsaro, Benoit Mosser and Masao Takata for comments on earlier drafts, which improved the manuscript considerably. The research leading to the presented results has received funding from the European Research Council under the European Community's Seventh Framework Programme (FP7/2007-2013)/ERC grant agreement no 338251 (StellarAges). YE acknowledges the support of the UK Science and Technology Facilities Council (STFC).

\section{References}

Bedding, T. R., Mosser, B., Huber, D., et al. 2011, Nature, 471, 608 Bossini, D., Miglio, A., Salaris, M., et al. 2015, MNRAS, 453, 2290 Bossini, D., Miglio, A., Salaris, M., et al. 2017, MNRAS, 469, 4718 Buysschaert, B., Beck, P. G., Corsaro, E., et al. 2016, A\&A, 588, A82

Campbell, S. W., \& Lattanzio, J. C. 2008, A\&A, 490, 769

Christensen-Dalsgaard, J. 2012, in Progress in Solar/Stellar Physics with Helio and Asteroseismology, eds. H. Shibahashi, M. Takata, \& A. E. Lynas-Gray, ASP Conf. Ser., 462, 503

Constantino, T., Campbell, S., Gil-Pons, P., \& Lattanzio, J. 2014, ApJ, 784, 56

Constantino, T., Campbell, S. W., Christensen-Dalsgaard, J., Lattanzio, J. C., \& Stello, D. 2015, MNRAS, 452, 123

Corsaro, E., Stello, D., Huber, D., et al. 2012, ApJ, 757, 190

Corsaro, E., De Ridder, J., \& García, R. A. 2015, A\&A, 579, A83

Cunha, M. S., Stello, D., Avelino, P. P., Christensen-Dalsgaard, J., \& Townsend, R. H. D. 2015, ApJ, 805, 127

Datta, A., Mazumdar, A., Gupta, U., \& Hekker, S. 2015, MNRAS, 447, 1935

Deheuvels, S., Ballot, J., Beck, P. G., et al. 2015, A\&A, 580, A96

Di Mauro, M. P., Ventura, R., Cardini, D., et al. 2016, ApJ, 817, 65

Goupil, M. J., Mosser, B., Marques, J. P., et al. 2013, A\&A, 549, A75

Hekker, S., \& Christensen-Dalsgaard, J. 2017, A\&ARv, 25, 1

Jiang, C., \& Christensen-Dalsgaard, J. 2014, MNRAS, 444, 3622

Lagarde, N., Bossini, D., Miglio, A., Vrard, M., \& Mosser, B. 2016, MNRAS, 457, L59

Lattanzio, J. C. 1986, ApJ, 311, 708

Mosser, B., Goupil, M. J., Belkacem, K., et al. 2012a, A\&A, 548, A10

Mosser, B., Goupil, M. J., Belkacem, K., et al. 2012b, A\&A, 540, A143

Mosser, B., Benomar, O., Belkacem, K., et al. 2014, A\&A, 572, L5

Mosser, B., Vrard, M., Belkacem, K., Deheuvels, S., \& Goupil, M. J. 2015, A\&A, 584, A50

Mosser, B., Pinçon, C., Belkacem, K., Takata, M., \& Vrard, M. 2017, A\&A, 600, A1

Paxton, B., Bildsten, L., Dotter, A., et al. 2011, ApJS, 192, 3

Silva Aguirre, V., Ruchti, G. R., Hekker, S., et al. 2014, ApJ, 784, L16

Takata, M. 2016a, PASJ, 68, 109

Takata, M. 2016b, PASJ, 68, 91
Unno, W., Osaki, Y., Ando, H., Saio, H., \& Shibahashi, H. 1989, Nonradial Oscillations of Stars, 2nd ed. (Tokyo, Japan: University of Tokyo Press) Vrard, M., Mosser, B., \& Samadi, R. 2016, A\&A, 588, A87

\section{Appendix A}

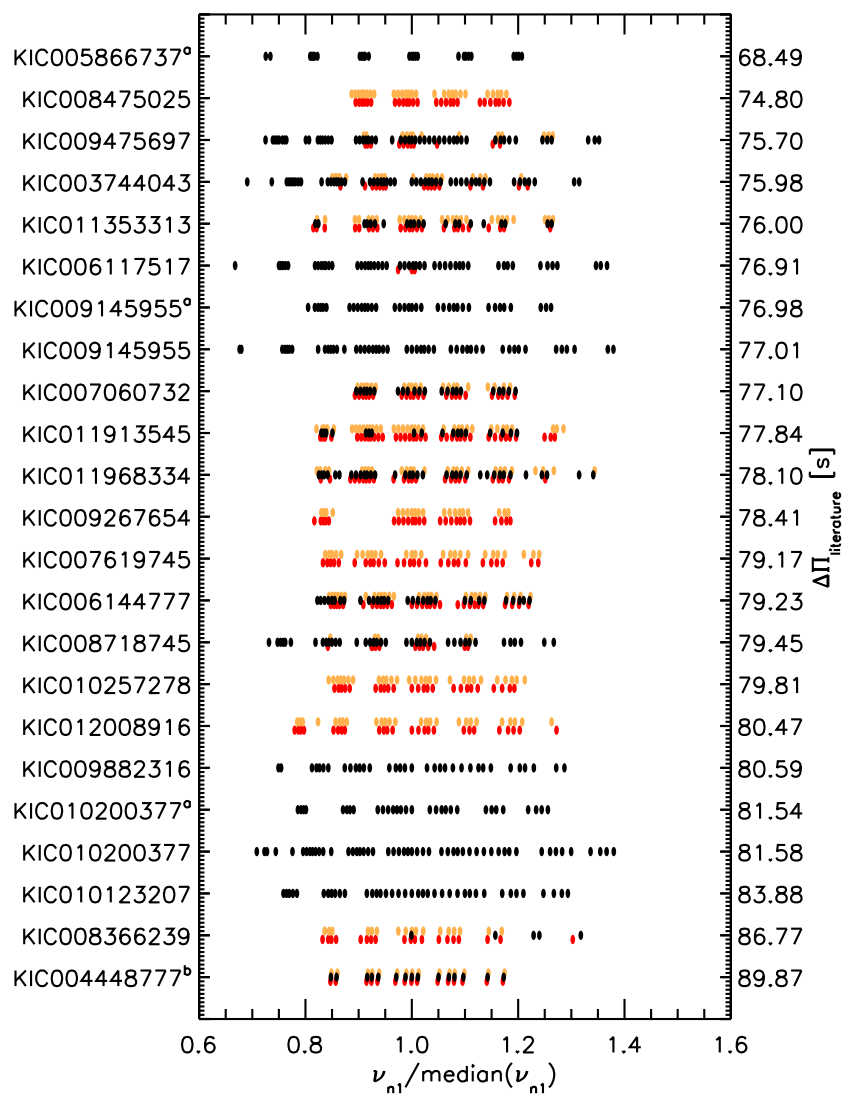

Fig. A.1. Dipole frequencies of all RGB stars used in the present analysis normalised by the median dipole frequency of the star. Modes with azimuthal orders $-1,0,1$ are shown (with a slight offset to one another) in red, black, and orange, respectively. Star names with a superscript "a" or "b" indicate that the data are taken from Datta et al. (2015) or Di Mauro et al. (2016), respectively. All other data are taken from Corsaro et al. (2015). On the left the period spacings presented in these references are shown (including the updates by Corsaro; see text). The stars are ordered following the RGB with the least-evolved star at the bottom and the most-evolved star at the top. 